\title{
Role of hypoxia-inducible factor in diabetic myocardial hypertrophy
}

\author{
L Qin ${ }^{1}$, S Xifa ${ }^{1}$, X Dawei ${ }^{1}$, X Yangjing ${ }^{1}$, J Kangting ${ }^{1}$, X Jian ${ }^{2}$, Z Suqin ${ }^{1}$ \\ ${ }^{1}$ The Second Affiliated Hospital of Wenzhou Medical University, ${ }^{2}$ Molecular Pharmacology Research Center, School of \\ Pharmacy, Wenzhou Medical University, Wenzhou Zhejiang 325000, PR China
}

*For correspondence: Email: zhangsuqinwz@163.com; Tel: +86-0577-88002213

\begin{abstract}
Purpose: This study was carried out to investigate the role of hypoxia-inducible factor (HIF) in diabetic cardiomyopathy in vitro.

Methods: Hypoxia was induced chemically in H9C2 cells (cardiac hypertrophy model), and the cells were treated with phenylephrine (PE), deferoxamine (DFO), PE + DFO, and HIF-1a siRNA under conditions of high and normal glucose. Western blot was used to analyze the expression of some glycolytic proteins, including Glut-1, hexokinase (HXK-2), and enolase, while apoptosis of H9C2 was determined by flow cytometry.

Results: PE caused hypertrophy in H9C2, which was ameliorated by HIF-1a. Compared to normal, under prolonged high glucose, the low expression of HIF-1a led to low expressions of Glut-1, HXK-2 and enolase. However, expression of HIF-1a decreased, while those of Bax and Caspase 3 increased, and $\mathrm{BCl}-2$ expression decreased. Furthermore, under short time high glucose, HIF-1a caused apoptosis of hypertrophic cardiomyocytes.

Conclusion: HIF-1 mediates diabetic myocardial hypertrophy, probably as a function of the degree of high glucose exposure and hypoxia.
\end{abstract}

Keywords: H9C2 cardiomyocyte, Hypoxia-inducible factor, Myocardial hypertrophy, Diabetic cardiomyopathy

Tropical Journal of Pharmaceutical Research is indexed by Science Citation Index (SciSearch), Scopus, International Pharmaceutical Abstract, Chemical Abstracts, Embase, Index Copernicus, EBSCO, African Index Medicus, JournalSeek, Journal Citation Reports/Science Edition, Directory of Open Access Journals (DOAJ), African Journal Online, Bioline International, Open-J-Gate and Pharmacy Abstracts

\section{INTRODUCTION}

Cardiac hypertrophy is one of the characteristic features of heart failure [1,2]. In cardiac hypertrophy, growth of cardiac tissue and maintenance of its function are associated with capillary angiogenesis. The survival of endothelial cells is mainly maintained by growth factors including vascular endothelial growth factor (VEGF) $[3,4]$, which plays an important role in the regulation of physiological and pathological processes in angiogenesis. It is known that specific knockout of VEGF can lead to decreased density of myocardial capillaries and systolic dysfunction [5,6]. In pathological cardiac hypertrophy, myocardial cells hold back the release of VEGF and bring down angiogenesis, thereby contributing to lack of oxygen and increased oxygen consumption. The hypoxia caused by this process firstly results in elevation of hypoxia inducible factor (HIF), which in turn leads to increases in levels of VEGF and other angiogenic factors. This adaptive response delays progression from pathological cardiac hypertrophy to heart failure. In early cardiac hypertrophy, stability of HIF-1 promotes glycolysis, which improves glucose utilization and 
allows for maintenance of normal functioning of the heart [7].

HIF is an important transcription factor involved in the regulation of about 300 genes which play important roles in the development of tissues and organs, proliferation and differentiation of cells, cellular energy metabolism, tumorigenesis and tumor progression $[8,9]$. HIF has three a subunits

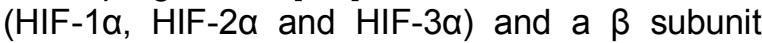
$[10,11]$. In general, the $\beta$ subunits in stable expression, and is usually polymerized with HIFa to form a heterodimer, which is transferred to the nucleus. HIF-1 is degraded through the ubiquitin-proteasome pathway, which process is mediated by the binding of HIF-1 and pVHL [12]. However, under hypoxic conditions, HIF-1 cannot be recognized by $\mathrm{pVHL}$, a situation which helps HIF escape from degradation and get transported to the nucleus for binding to hypoxia response element (HRE) and the coactivator p300. Then the transcription of HIF-1 is initiated to offer the molecular foundation of the rapid response to hypoxia. It has been reported that HIF-1a expression in patients with ischemic heart disease is increased, whereas systemic knockout of HIF-1a can lead to cardiac hypertrophy and even death of embryos. Moreover, cardiacspecific knockout of HIF-1a can bring about changes in angiogenesis, energy availability, calcium flux and cardiac systolic function under normal oxygen conditions [13].

A large number of reports indicate that diabetes is closely related to angiogenesis. Diabetes or hyperglycemia disrupts HIF-mediated cardiac hypertrophy adaptive regulatory mechanism [14]. In diabetic retinopathy, abnormal increase of angiogenesis is directly related to elevated VEGF expression [15]. However, reduced expression of VEGF and its receptor in people with diabetic trauma, induces insufficient angiogenesis $[16,17]$.

Cardiac hypertrophy caused by cardiac overload increases oxygen consumption, but when the amount of oxygen is reduced, it results in cardiac hypoxia. This process accelerates the activation of HIF-1, resulting in increase in levels of VEGF and other angiogenic factors and enhancement of glucose metabolism, which promotes angiogenesis and energy metabolism. These events delay the progression from cardiac hypertrophy to heart failure. So far, many studies have shown that diabetes is harmful to the heart. Short-term hyperglycemia may promote HIF-1 activation, which in turn increases the expression of VEGF, HXK-2, Glut-1 and enolase [18], but long-term hyperglycemia damages the function of HIF-1.
The role of HIF in diabetic cardiac hypertrophy is essentially unexplored. This study was carried out to investigate if HIF plays any role in diabetic cardiomyopathy in vitro.

\section{METHODS}

\section{Induction of cardiac hypertrophy}

Cardiac hypertrophy was induced in $\mathrm{H} 9 \mathrm{C} 2$ cardiomyocytes with phenylephrine (PE) (Sigma) [19], and deferoxamine (DFO) (Sigma) was used to induce the expression of HIF. HIF-1 $\alpha$ was silenced with siRNA technology. The cells were cultured in normal-sugar medium and high-sugar medium (Invitrogen). Under three states of normal glucose (NG), high glucose (HG) and HIF1- $\alpha$ silence the expression of HIF-1 $\alpha$, HIF-2 $\alpha$, Glut-1, enolase, HXK-2, Bax and Bcl-2 in H9C2 cardiomyocytes were measured [20], as well as apoptosis of the H9C2 cells.

\section{Cell line and culture}

We used H9C2 cardiomyocytes from ATCC. The cells were cultured in DMEM containing $10 \%$ heat-inactivated fetal bovine serum and $1 \%$ penicillin-streptomycin mixture, and were grown at $37{ }^{\circ} \mathrm{C}$ under a humidified atmosphere of $5 \%$ $\mathrm{CO}_{2}$ and $95 \%$ air.

\section{HIF-1 $\alpha$ silencing}

We dissolved 200 pM siRNA in a selective medium MEM, which was mixed with liposome 2000 for 25 minutes at room temperature. This was then added to each well of a six-well plate containing $5 \times 10^{5}$ H9C2 cardiomyocytes, and incubated for 4-6 h. Following that, antibiotic-free DMEM medium was replaced after $24 \mathrm{~h}$ incubation.

\section{Western blot analysis}

$\mathrm{PE}, \mathrm{PE}+\mathrm{DFO}, \mathrm{PE}+\mathrm{DFO}$ and HIF-1 1 siRNA were separately used to treat the $\mathrm{H} 2 \mathrm{C} 9$ cardiomyocytes under conditions of high glucose (HG) and normal glucose (NG) for $48 \mathrm{~h}$. The cells were harvested and lysed in RIPA buffer with protease inhibitors. Protein concentration was determined by the Coomassie brilliant blue method. Equal amounts of total cell lysates (60 mg protein) and Full-Range Rainbow Molecular Weight Marker were resolved using $12 \%$ sodium dodecyl sulfate polyacrylamide gel electrophoresis (SDS-PAGE) and transferred onto polyvinylidene fluoride (PVDF) membranes through electrophoresis. PVDF membranes were immersed in blocking solution (5\% skim milk) to block non-specific binding for $1 \mathrm{~h}$ at room 
temperature. Thereafter, the PVDF membranes were immuno-blotted with primary monoclonal antibodies at 1:300 dilution at room temperature for $2 \mathrm{~h}$, then overnight at $4{ }^{\circ} \mathrm{C}$. Following this, further incubation was conducted for $2 \mathrm{~h}$ at room temperature with rabbit anti-mouse horseradish peroxide (HRP)-conjugated secondary antibody (1:3000 dilution). The protein-antibody complex was detected by enhanced chemiluminescence detection system.

\section{Flow cytometry}

After digesting with EDTA-free trypsin, the H9C2 cells were centrifuged at $1000 \mathrm{rpm}$ for $5 \mathrm{~min}$. After discarding the medium, the cells were washed three times with PBS. Thereafter an appropriate amount of binding buffer was added to achieve a dilution resulting in a concentration of $10^{5}$ cells per $100 \mu \mathrm{L}$. In the dark, $5 \mu \mathrm{L}$ Annexin $\mathrm{V}$-FITC and $3 \mu \mathrm{L} \mathrm{PI}$ were added to $100 \mu \mathrm{L}$ cells and stained for $10 \mathrm{~min}$. Following that, $400 \mu \mathrm{L}$ binding buffer was added. The fluorescence intensity of FITC was determined $(\lambda \mathrm{Ex} / \mathrm{Em}=$ $488 / 515 \mathrm{~nm})$, and apoptosis of cells was determined by fluorescence intensity of $\mathrm{PI}(\lambda=$ 560nm).

\section{Statistical analysis}

Experimental data are expressed as mean \pm standard deviation (SD) after quantitation or semi-quantitation. Comparisons were analyzed using one-way analysis of variance (ANOVA). $P$ $<0.05$ was taken as statistically significant. Statistical analysis was performed using SPSS 17.0 while plots were made with GraphPad Prism5.

\section{RESULTS}

\section{Phenylephrine-induced hypertrophy of H9C2}

Under an optical microscope (Figure 1), the control displayed normal features of $\mathrm{H} 9 \mathrm{C} 2$ cells, with spindle shape, round and large-centered nucleus and small cell interval. $\mathrm{H} 9 \mathrm{C} 2$ cardiomyocytes incubated with PE for $48 \mathrm{~h}$ were much larger and circular, round or elliptical in shape. Moreover, significant expansion of the cytoplasm, large cell interval and bigger nucleus were observed.

\section{HIF-1a relieved cardiomyocytes hypertrophy}

After culturing in $1 \%$ serum medium for $12 \mathrm{~h}$, H9C2 cells were treated with PE $(100 \mu \mathrm{M})$ and DFO $(200 \mu \mathrm{M})$ respectively for $48 \mathrm{~h}$. Then HIF-1a gene was silenced with liposome 2000. Under an optical microscope (Figure 2), in the case of NG, $\mathrm{PE}$ caused mild hypertrophy of myocardial cells, which was relieved after adding DFO to induced HIF-1a. In the HG group, cardiomyocytes treated with PE exhibited obvious myocardial hypertrophy, which was not mitigated by adding DFO due to HG-blockage of HIF-1a activation. Moreover, the hypertrophy in myocardial cells with silenced HIF-1a was almost unchanged in morphology.

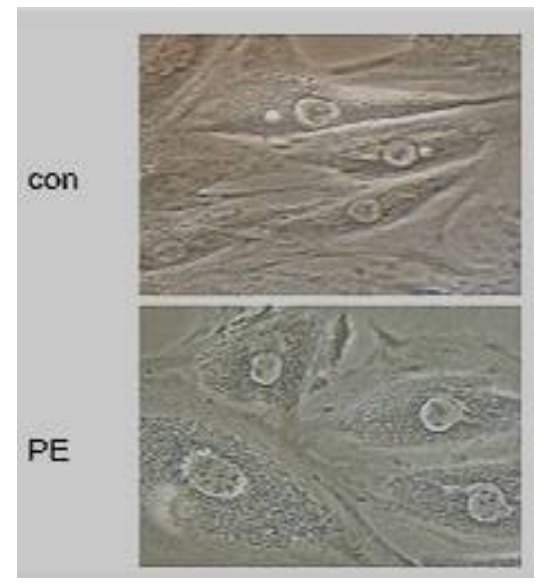

Figure 1: PE-induced hypertrophy of $\mathrm{H} 9 \mathrm{C} 2$ cardiomyocytes. From top to bottom are normal cardiomyocytes, cardiomyocytes treated with PE, respectively

\section{Effect of HIF-1a on expression of proteins associated with glycolysis}

As shown in Figure 3, silencing of HIF-1a gene resulted in reduced expression of Glut-1, HXK-2 and enolase. In the NG and HG groups, myocardial hypertrophy occurred after treatment with $\mathrm{PE}$, and in the groups administered with DFO, HIF-1a expression was relatively higher than in the groups treated with $P E$ only. In addition, SiRNA reduced the expression of HIF1a. HG decreased the activation of HIF-1a, which led to lower expression of HIF-1a in this group than in the NG group. Administration of DFO to the HG group resulted in corresponding decrease in the expression of Glut-1. Long-term HG treatment led to decreased expression of HXK-2 but treatment with DFO increased the expressions of HXK-2 and enolase.

\section{Effect of HIF-1a on the expression of proteins associated with apoptosis}

As shown in Figure 4, compared with the normal group, HIF expression was reduced, while Bax increased, Bcl-2 decreased and activated caspase 3 was higher in the long-term HG group. 


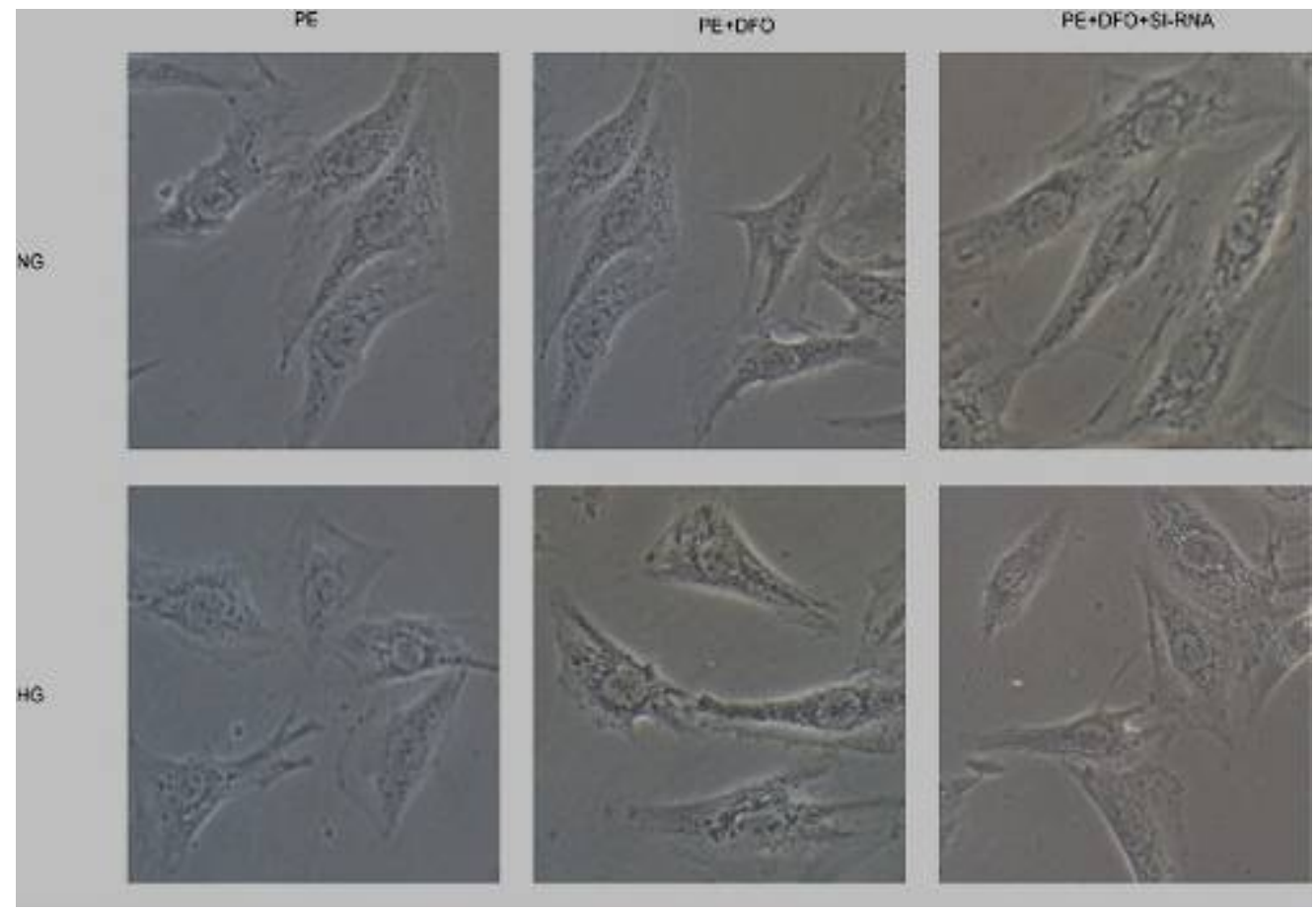

Figure 2: Relief of cardiomyocyte hypertrophy by HIF-1a (NG is the group treated with normal glucose medium; $H G$ is the high glucose medium; PE was treated t with PE only; PE + DFO group received simultaneously PE and DFO ; PE + DFO + Si-RNA group got PE and DFO simultaneously for 48 hours, prior to silencing the HIF-1 $\alpha$ genome with liposome 2000)

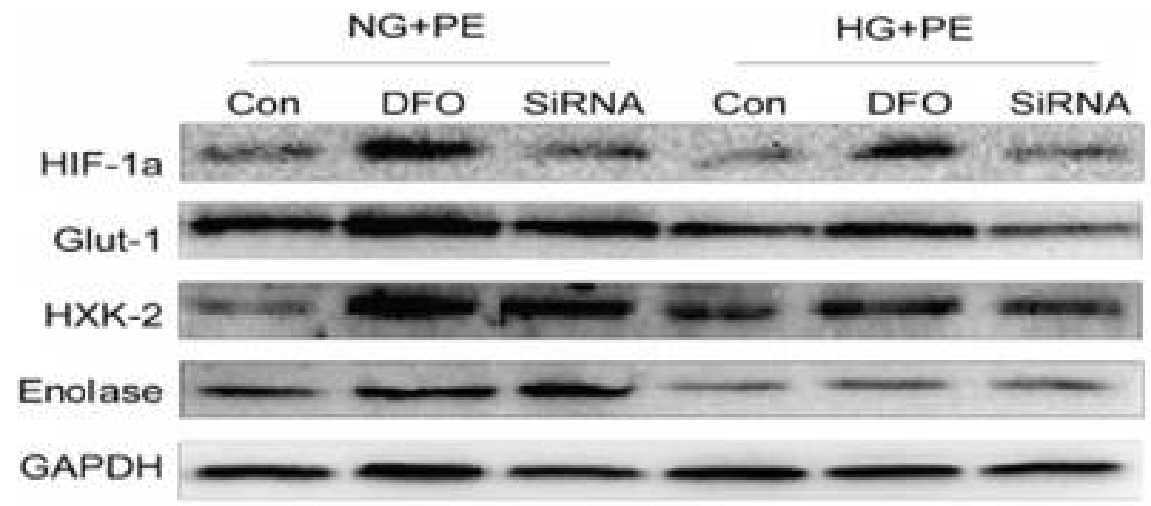

Figure 3: Effect of PE on HIF-1 $\alpha$ induced reduction in expression of Glut-1, HXK-2 and enolase under normal glucose (NG) and high glucose (HG)

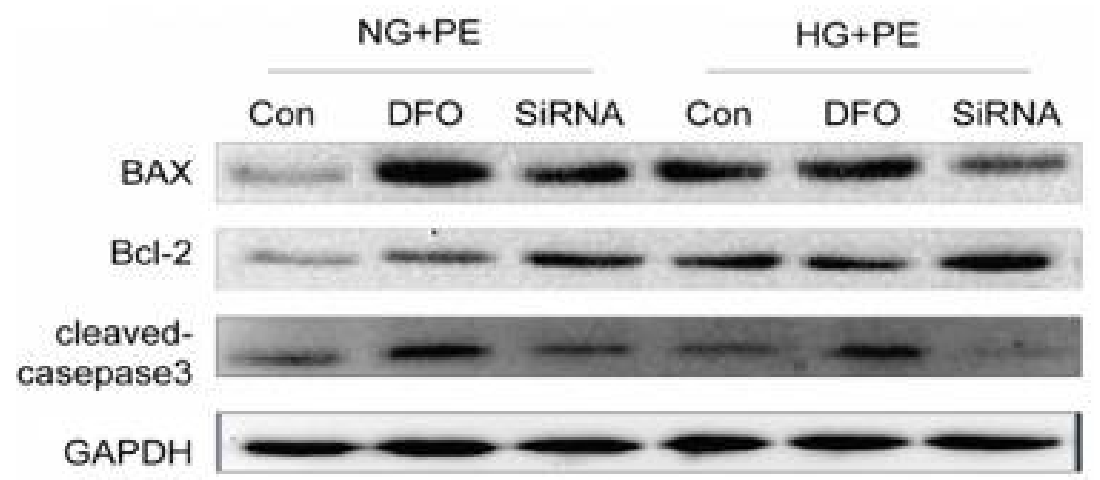

Figure 4: Effect of PE under normal glucose (NG) and high glucose (HG) on expression of apoptosis-related proteins in $\mathrm{H} 9 \mathrm{C} 2$ cells 

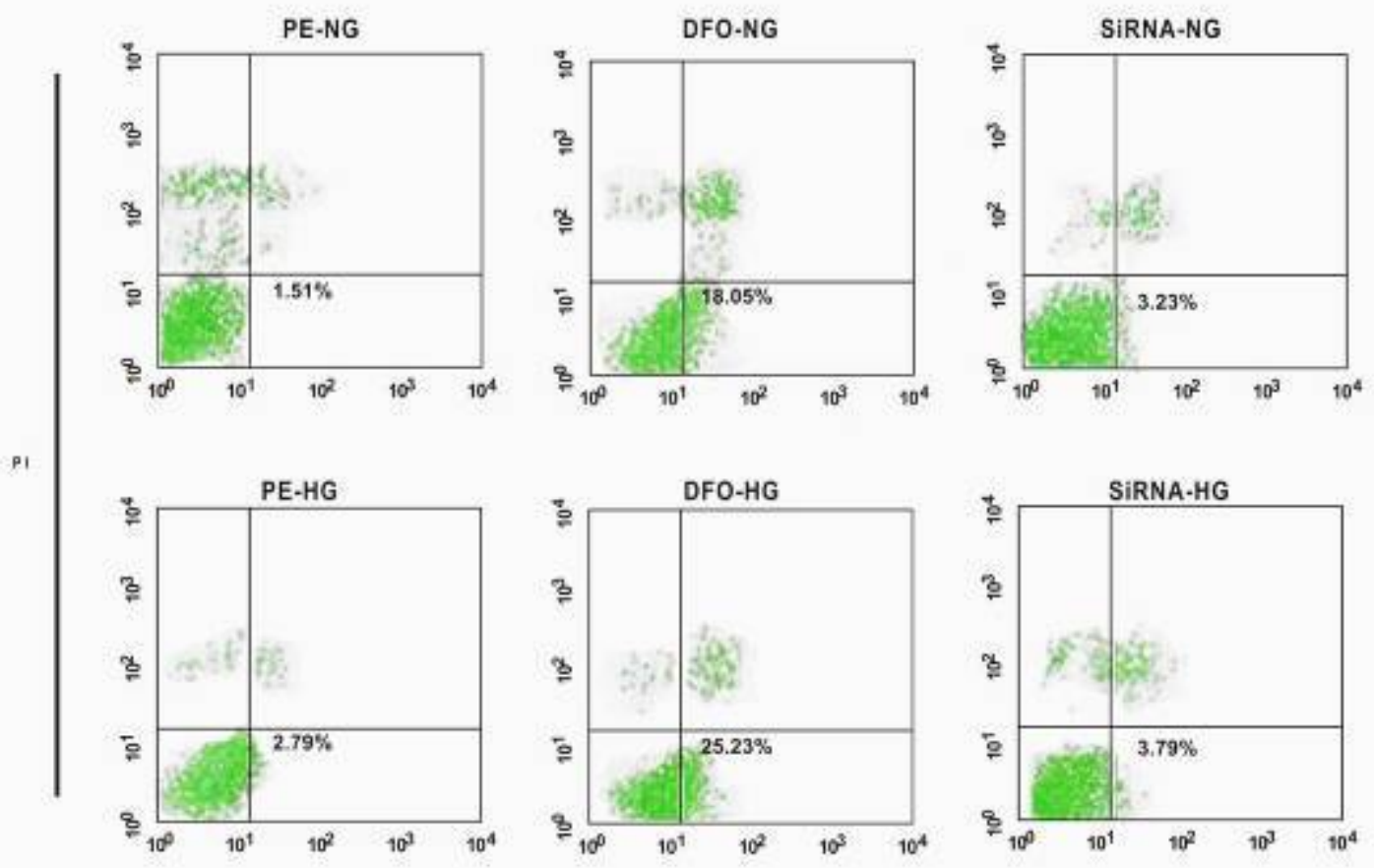

Annoxin V-FITC

Figure 5: HIF-1a mediated cell apoptosis in diabetic myopathy. The lower left corner of each box represents living cells. Cells in the lower left corner, top right corner and lower right corner are living cells, necrotic cells and early apoptotic cells, respectively

\section{Evidence of apoptosis from flow cytometry}

Results from flow cytometry are shown in Figure 5. HIF-1a mediated the apoptosis of the hypertrophic cardiomyocytes exposed to shortterm high glucose concentrations.

\section{DISCUSSION}

Previous studies have shown that high expression of heart HIF-specific transgene prevented myocardium structural disorder and myocardial fibrosis in mice and exhibited the decrease of VEGF, HXK-2 and Glut-1 caused by diabetes [18]. Enhanced HIF-1a promotes antioxidant capacity of the heart and prevents the occurrence of diabetic cardiomyopathy. However, cardiac hypertrophy associated with pressure lowers HIF-1 $\alpha$ [7]. Cell culture studies have demonstrated that high glucose conditions block HIF-1 $\alpha$ activation, thereby blocking adaptation to hypoxia. The current study is based on earlier investigations on the role of HIF in myocardial hypertrophy under high glucose conditions in vitro cell culture experiments $[19,21,22]$. We used PE to establish the cardiac hypertrophy model, and DFO was given to mimic a hypoxic environment necessary for HIF production. Moreover, gene silencing technology was used to silence HIF-1a. Under NG, the PEtreated cells displayed slight hypertrophy, but after treatment with DFO, cell morphology was restored. While HG made myocardial hypertrophy more obvious, even DFO-induced HIF-1a did not significantly mitigate the hypertrophy. This is consistent with previous studies stating that high glucose is detrimental to the activity of HIF [18]. HIF-1a silencing did not change cell morphology substantially, which further indicates that HIF-1 $\alpha$ has a protective effect on myocardial hypertrophy [18].

In the pathogenesis of heart failure, as well as the physiological function of blood vessels, significantly altered glucose and lipid metabolism is associated with heart failure [14]. The transition from aerobic metabolism to glycolysis is one of the key cellular processes for adaption to hypoxia response, which is regulated by HIF1. Therefore, we examined the expression of glycolytic proteins using Western Blot. The decrease in HIF-1a resulted in reduced expression of Glut-1, HXK-2 and enolase. Hypoxia can easily induce apoptosis, so it was necessary to study the expressions of apoptosisrelated proteins as well. The results obtained showed that Bax expression was increased, while that of $\mathrm{Bcl}-2$ decreased, and activated 
caspase 3 was higher under long-term high glucose condition. The results suggest that high glucose levels impair HIF activity, and that HIF protected the $\mathrm{H} 9 \mathrm{C} 2$ cells from apoptosis.

However, results of flow cytometry indicated that HIF-1 promoted cell apoptosis under short-term high glucose condition, which is at variance with the western blot results on apoptosis-associated proteins. The reason for this apparent contradiction is not very clear, but it may be related to degree of high glucose exposure and hypoxia.

\section{CONCLUSION}

Short-term hyperglycemia may contribute to activation of HIF-1, which in turn increases the expression of VEGF, HXK-2 and Glut-1. However, long-term high blood sugar may be detrimental to the function of HIF-1. It can be inferred from our results that HIF-1 is likely to play an important role in diabetic cardiac hypertrophy. On account of different effects of HIF-1 under different glucose loads and hypoxia, it is concluded that HIF may play a pathogenic role in heart failure.

\section{DECLARATIONS}

\section{Acknowledgement}

This work was supported by a grant from Zhejiang Provincial Science Foundation (no. LY13H020004). This manuscript has not been previously published, nor is it before another journal for consideration.

\section{Conflict of Interest}

No conflict of interest associated with this work.

\section{Contribution of Authors}

The authors declare that this work was done by the authors named in this article and all liabilities pertaining to claims relating to the content of this article will be borne by them. Suqin $Z$ conceived and designed the study. Qin L, Xifa S, Dawei X, Yangjing $X$, Kangting $J$ and Jian $X$ collected the data. Qin $L$ and Xifa $S$ analyzed the data.

\section{Open Access}

This is an Open Access article distributed under the terms of the Creative Commons Attribution License, which permits unrestricted use, distribution, and reproduction in any medium, provided the original work is properly credited.

\section{REFERENCES}

1. Shiojima I, Sato K, Izumiya $Y$, Schiekofer S, Ito M, Liao $R$, Colucci WS, Walsh K. Disruption of coordinated cardiac hypertrophy and angiogenesis contributes to the transition to heart failure. J Clin Investig 2005; 115(8): 2108-2118.

2. Gerdes AM, Kellerman SE, Moore JA, Muffly KE, Clark LC, Reaves PY, Malec KB, McKeown PP, Schocken $D D$. Structural remodeling of cardiac myocytes in patients with ischemic cardiomyopathy. Circ 1992; 86(2): 426-430.

3. Gao W, Ferguson G, Connell P, Walshe T, Murphy $R$, Birney YA, O'Brien $C$, Cahill $P A$. High glucose concentrations alter hypoxia-induced control of vascular smooth muscle cell growth via a HIF-1alpha-dependent pathway. J Mol Cell Cardiol 2007; 42(3): 609-619.

4. Walsh K, Shiojima I. Cardiac growth and angiogenesis coordinated by intertissue interactions. J Clin Investig 2007; 117(11): 3176-3179.

5. Giordano FJ, Gerber HP, Williams SP, Vanbruggen $N$, Bunting S, Ruizlozano $P, G u Y$, Nath AK, Huang $Y$, Hickey R. A cardiac myocyte vascular endothelial growth factor paracrine pathway is required to maintain cardiac function. Proc Natl Acad Sci 2001; 98(10): 57805785.

6. Brown MD, Hudlická O. Angiogenesis in Skeletal and Cardiac Muscle. Physiol Rev 1992; 72(2): 369-417.

7. Jiang $Y$, Reynolds $C, C$, Feng $W$, Zhou Z, Rodriguez $W$, Tyagi S, Eaton J, Saari J, Kang Y. Dietary copper supplementation reverses hypertrophic cardiomyopathy induced by chronic pressure overload in mice. $J$ Exp Med 2007; 204(3): 657-666.

8. Wang GL, Jiang BH, Rue EA, Semenza GL. Hypoxiainducible factor 1 is a basic-helix-loop-helix-PAS heterodimer regulated by cellular $\mathrm{O} 2$ tension. Proc Natl Acad Sci 1995; 92(12): 5510-5514.

9. Huang LE, Gu J, Schau M, Bunn HF. Regulation of hypoxia-inducible factor 1alpha is mediated by an O2dependent degradation domain via the ubiquitinproteasome pathway. Proc Natl Acad Sci U S A 1998; 95(14): 7987-7992.

10. Epstein ACR, Gleadle JM, Mcneill LA, Hewitson KS, O'Rourke J, Mole DR, Mukherji M, Metzen E, Wilson MI, Dhanda A. C. elegans EGL-9 and Mammalian Homologs Define a Family of Dioxygenases that Regulate HIF by Prolyl Hydroxylation. Cell 2001; 107(1): 43-54.

11. Maxwell PH, Wiesener MS, Chang GW, Clifford SC, Vaux EC, Cockman ME, Wykoff CC, Pugh CW, Maher $E R$, Ratcliffe PJ. The tumour suppressor protein VHL targets hypoxia-inducible factors for oxygen-dependent proteolysis. Nat 1999; 399(6733): 271-275.

12. Lando D, Peet DJ, Whelan DA, Gorman JJ, Whitelaw ML. Asparagine Hydroxylation of the HIF Transactivation Trop J Pharm Res, November 2016; 15(11): 2426 
Domain: A Hypoxic Switch. Sci 2002; 295(5556): 858861.

13. Huang Y, Hickey RP, Yeh JL, Liu D, Dadak A, Young LH, Johnson RS, Giordano FJ. Cardiac myocyte-specific HIF-1alpha deletion alters vascularization, energy availability, calcium flux, and contractility in the normoxic heart. FASEB J 2004; 18(10): 1138-1140.

14. Renard $C B$, Kramer $F$, Johansson $F$, Lamharzi $N$, Tannock LR, von Herrath MG, Chait A, Bornfeldt KE. Diabetes and diabetes-associated lipid abnormalities have distinct effects on initiation and progression of atherosclerotic lesions. J Clin Investig 2004; 114(5): 659-668.

15. Wilkinson-Berka JL. Vasoactive factors and diabetic retinopathy: vascular endothelial growth factor, cycoloxygenase-2 and nitric oxide. Curr Pharm Des 2004; 10(27): 3331-3348.

16. Galiano RD, Tepper OM, Pelo CR. Topical vascular endothelial growth factor accelerates diabetic wound healing through increased angiogenesis and by mobilizing and recruiting bone marrow-derived cells. Am J Pathology 2004; 164(6): 1935-1947.

17. Chou E, Suzuma I, Way KJ, Opland D, Clermont AC, Naruse K, Suzuma K, Bowling NL, Vlahos CJ, Aiello LP. Decreased cardiac expression of vascular endothelial growth factor and its receptors in insulin-resistant and diabetic States: a possible explanation for impaired collateral formation in cardiac tissue. Circ 2002; 105(3): 373-379.

18. Xue W, Cai LY, Thistlethwaite $P$, Kang YJ, Li X, Feng W. Cardiac-specific overexpression of HIF-1\{alpha\} prevents deterioration of glycolytic pathway and cardiac remodeling in streptozotocin-induced diabetic mice. Am J Pathology 2010; 177(1): 97-105.

19. Clerk A, Sugden PH. Activation of protein kinase cascades in the heart by hypertrophic $G$ proteincoupled receptor agonists. Am J Cardiol 1999; 83(12A): 64-69.

20. Liu Q, Wang G, Zhou G, Tan Y, Wang X, Wei W, Liu L, Xue $W$, Feng $W$, Cai $L$. Angiotensin II-induced p53dependent cardiac apoptotic cell death: Its prevention by metallothionein. Toxicol Lett 2009; 191(2-3): 314-320.

21. Ceriello A, Quagliaro L, D'Amico M, Di FC, Marfella R, Nappo F, Berrino L, Rossi F, Giugliano D. Acute hyperglycemia induces nitrotyrosine formation and apoptosis in perfused heart from rat. Diabetes 2002; 51(4): 1076-1082.

22. Ma G, Al-Shabrawey M, Johnson JA, Datar R, Tawfik HE, Guo D, Caldwell RB, Caldwell RW. Protection against myocardial ischemia/reperfusion injury by short-term diabetes: enhancement of VEGF formation, capillary density, and activation of cell survival signaling. Eur $J$ Pharmacol 2006; 373(6): 415-427. 